\title{
Viewpoint: experimental recovery of geometrically necessary dislocation density in polycrystals
}

\author{
Brent L. Adams \\ b_I_adams@byu.edu \\ Bassem S. El-Dasher \\ Anthony D. Rollett
}

Follow this and additional works at: https://scholarsarchive.byu.edu/facpub

Part of the Mechanical Engineering Commons

Original Publication Citation

Scripta Materialia, Volume 48, Number 2, January 23, pp. 141-145(5)

\section{BYU ScholarsArchive Citation}

Adams, Brent L.; El-Dasher, Bassem S.; and Rollett, Anthony D., "Viewpoint: experimental recovery of geometrically necessary dislocation density in polycrystals" (2002). Faculty Publications. 535. https://scholarsarchive.byu.edu/facpub/535 


\title{
Viewpoint: experimental recovery of geometrically necessary dislocation density in polycrystals
}

\author{
B.S. El-Dasher ${ }^{\mathrm{a}, *}$, B.L. Adams ${ }^{\mathrm{b}}$, A.D. Rollett ${ }^{\mathrm{a}}$ \\ ${ }^{a}$ Materials Science and Engineering Department, Carnegie Mellon University, Pittsburgh, PA 15206, USA \\ ${ }^{\mathrm{b}}$ Mechanical Engineering Department, Brigham Young University, Provo, UT 84602, USA
}

\begin{abstract}
Application of electron backscattering diffraction methods to recover estimates of the geometrically necessary dislocation density is described. The limitations of the method arising from the opacity of crystalline materials and the spatial and angular resolution limits are discussed.
\end{abstract}

Keywords: Dislocations; Aluminum; Grain Boundaries; Electron backscattering diffraction

\section{Introduction}

It has long been an experimental observation that the constituent grains of polycrystalline materials develop complex patterns of heterogeneity over a wide range of length scales during plastic deformation. The classical theory of crystal plasticity is limited to those interactions arising from the enforcement of mechanical compatibility [1]. It is evident that an understanding of the behavior of grain boundaries, and how they interact with deformation mechanisms as they change their structure to accommodate deformation, has been missing. These interactions are anticipated to set the necessary length scale(s) seen clearly in the Hall-Petch relationship and as discussed in the

\footnotetext{
${ }^{*}$ Corresponding author. Tel.: +1-412-519-5555; fax: +1-412363-0182.

E-mail address: be25@andrew.cmu.edu (B.S. El-Dasher).
}

context of strain gradient plasticity [2]. Recent work has shown that an experimental technique may help provide this length scale by utilizing information obtained by electron backscattering diffraction (EBSD). Orientation imaging microscopy (OIM) provides automated scanning measurements of the lattice curvature near grain boundaries. Relating this curvature to estimates of the geometrically necessary dislocation densities (GNDs) provides a promising experimental vehicle for the study of plasticity in crystalline materials [3]. The purpose of this paper is to discuss the strengths and limitations of this method. The principal limitations of the OIM-based methods pertain to material opacity and the spatial/angular resolution of EBSD. In the sections that follow italics are used to emphasis strengths and weaknesses of these methods. 


\section{Theory}

From a continuum perspective, GNDs are those that are required to support a particular curvature in the crystallographic lattice at any given point in a deformed structure [4]. The fundamental equation of continuum dislocation theory establishes a link between the elastic distortion tensor $\beta^{e}$ and the dislocation tensor $\alpha$ [5]:

$\alpha=\operatorname{curl} \beta^{e}$.

This can be simplified to a form that relates the dislocation tensor to the curvature of the elastic strain and lattice orientation [3]:

$\alpha_{i j}=e_{i k l}\left(\varepsilon_{j l, k}^{e}+g_{j l, k}\right)$,

where $e_{i k l}$ are components of the permutation tensor, $\varepsilon_{j l, k}^{e}$ the infinitesimal elastic strain gradient, and $g_{j l, k}$ the gradient in lattice orientation. In the absence of long-range elastic stress fields, Nye's original formulation of the dislocation tensor is retrieved [6]:

$\alpha_{i j}=e_{i k l} g_{j l, k}$,

thus, providing a direct relationship between the measured crystallographic orientation gradient and the dislocation tensor. Nye's original work also shows a precise connection between the dislocation tensor and the local dislocation network, formally expressed as:

$\alpha_{i j}=\sum_{k=1}^{K} \rho^{k} b_{i}^{k} \hat{z}_{j}^{k}$,

where the sum is over all the dislocation types present in the material, $\rho^{k}$ denotes the density of the dislocation of type $k$, and $\mathbf{b}^{k}$ and $\hat{z}^{k}$ denote the Burger's vector and unit line direction of the specific dislocation type. Nye originally solved this relation for the trivial case of the dislocation systems of a simple cubic lattice. Application of Eq. (4) to materials with other crystal structures is nontrivial in that no unique solution is possible; lowerbound estimates on dislocation densities have been obtained [3,7,8].

Note that Frank's analysis for low-angle boundaries can also be used to calculate the required dislocation densities between neighboring points. While it does assume that the orientation gradient is collapsed into a plane between the points, it is fundamentally the same in that one recovers a surface dislocation at a particular limit [9].

\section{Current experimental methods}

Here we describe recent experimental work carried out on cylindrical samples cut from a directionally solidified, high purity $(5 \mathrm{~N})$ aluminum ingot. Samples were cut so their cylindrical axis was parallel to the solidification axis. The resulting microstructure consisted of long, columnar grains with the $\langle 001\rangle$ crystallographic axis parallel to the columnar direction and diameters between 1 and 3 $\mathrm{mm}$. The columnar nature of the sample permits the critical assumption that the lattice curvature into the characterized plane of the sample is negligible relative to the in-plane curvature: $g_{j l, 3} \cong 0$. The principal limitation being addressed in the preparation of columnar microstructures is the electron opacity of crystalline materials. It is known that backscattered electrons interact only with the first $\sim 20 \mathrm{~nm}$ of the surface, and therefore experimental determination of gradient terms of the form $g_{j l, 3}$ is not feasible. This is the fundamental problem of crystalline opacity. Given that only components of the form $g_{j l, 1}$ and $g_{j l, 2}$ are experimentally accessible by electron diffraction, unless $g_{j l, 3}=0$ it is only possible to recover three of the nine components $\alpha_{i j}$ of the dislocation tensor. In principle of course, serial sectioning could be employed to characterize orientation in the third dimension. However, besides the obvious challenges of sectional alignment, the resolution limits in the third dimension are very poor compared to inplane resolutions.

Uniaxial compression tests were performed at set strain levels, with the compression axis parallel to the cylindrical axis of the sample. After specimen preparation, spatial crystallographic orientation information was then obtained near high angle grain boundaries using an automated OIM system coupled to a FEGSEM. Orientation information was collected about grain boundaries in a square grid with $500 \mathrm{~nm}$ steps. Typical grid sizes were on the order of $200 \times 200 \mu \mathrm{m}^{2}$. Using similar 
methods to those previously reported [10], the accuracy of orientation measurement was found to be $\approx 5 \%$ for the presented results. Typically, we find that under the best conditions, an angular precision of $\sim 0.01$ is achieved in modern FEGSEM instruments. Spatial resolution is strongly dependent upon the molecular weight of the crystalline material, and is typically not better than $\sim 20 \mathrm{~nm}$.

\section{Data analysis and visualization}

Datasets obtained from compressed samples were examined in the context described above using the physically-restricted lower-bound fcc deconstruction described in [8].

The 24 proper symmetry operators for cubic crystal symmetry were applied to every point in the

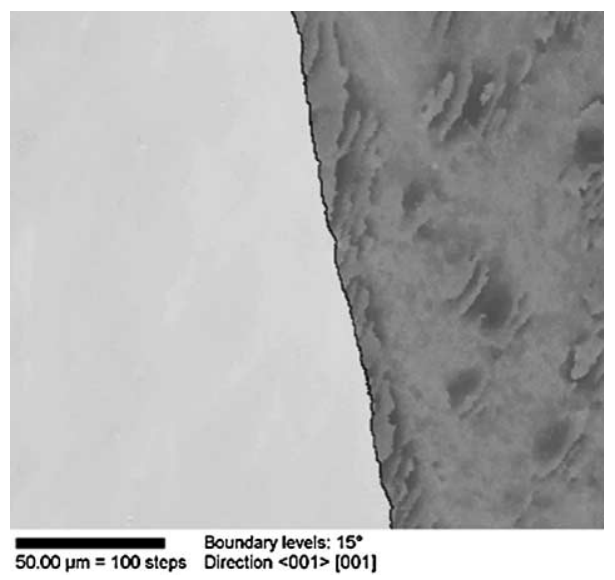

Fig. 1. Crystal orientation plot. Lighter grayscale indicates orientation closer to crystallographic $\langle 001\rangle$.
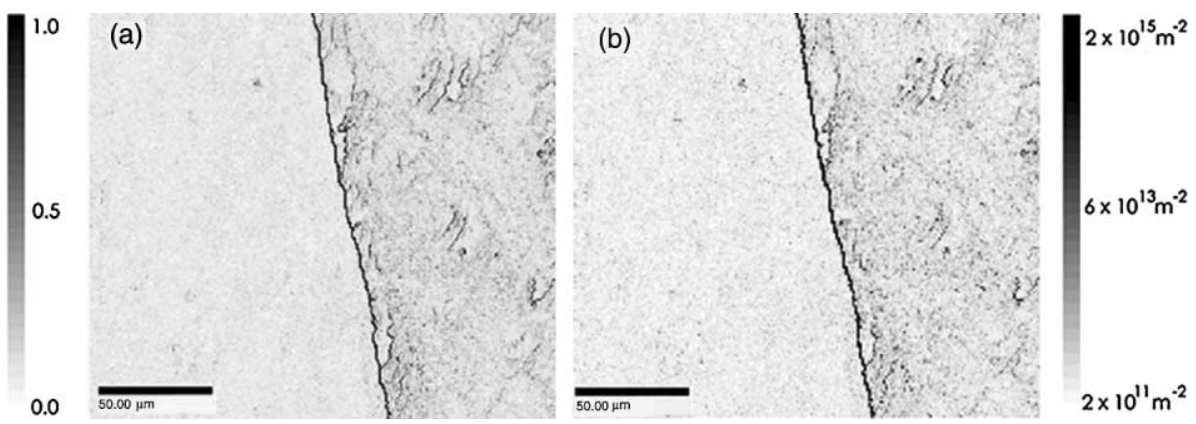

Fig. 2. Intensity plots of the magnitude of (a) the magnitude of the dislocation tensor and (b) $\rho_{\mathrm{GND}}$. dataset before the computation, to insure that all measured gradients were true. The issue here is that 24 orientation tensors represent the same crystal orientation, and thus care must be taken in calculating orientation gradients to insure congruence. Fig. 1 illustrates a crystal orientation map obtained from a typical dataset (in this case from a sample deformed to $\approx 12 \%$ strain) where darker grayscales indicate a larger deviation of the $\langle 001\rangle$ crystal axis from the sample surface normal. It can be readily seen that in this region, the orientation variations are larger in the grain to the right of the boundary (grain 2), as opposed to those in the grain to the left (grain 1). This can be misleading, however, because this map does not contain any information about in-plane orientation.

A more complete visualization of lattice curvature is necessary, especially if one is to successfully identify the interaction length scales. To this end, two-dimensional intensity plots are created to highlight the local lattice curvature as seen in Fig. $2 \mathrm{a}$, where the intensity presented is the sum of the magnitude of the dislocation tensor components:

$$
\alpha^{\text {total }}=\sum_{i=1}^{3} \sum_{j=1}^{3}\left|\alpha_{i j}\right|
$$

Note that this allows for the direct visualization of the locations of the greatest orientation curvature (in this case observed in grain 2, as well as the other less obvious "pockets" of orientation curvature in grain 1). A natural extension of this, shown in Fig. 2b, is the 2-D plot of total GND density, $\rho_{\mathrm{GND}}$, which is defined by: 


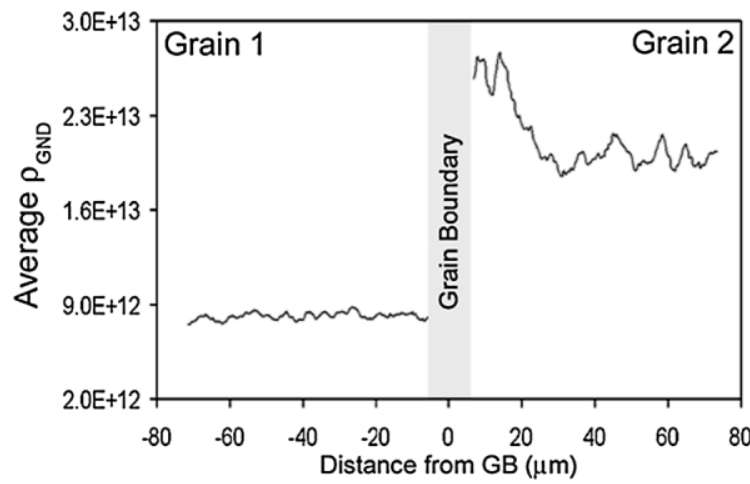

Fig. 3. Plot of the average $\rho_{\mathrm{GND}}$ as a function of the distance from the grain boundary.

$\rho_{\mathrm{GND}}=\sum_{k^{\prime \prime} \in D^{\prime \prime}} \rho^{k^{\prime \prime}}$.

Here $D^{\prime \prime}$ denotes the subset of dislocations obtained from the physically-restricted fcc deconstruction, and includes the nine most highly stressed dislocation systems (from the full set of 36), on the basis of the Schmid factor [8]. As expected, a direct correlation can be made between the $\alpha^{\text {total }}$ and $\rho_{\mathrm{GND}}$ plots at any given point.

Fig. 3 shows typical results when averages of $\rho_{\mathrm{GND}}$ are obtained as a function of distance from the boundary. OIM-based methodology has been remarkable in showing long-range accumulations of GNDs near grain boundaries in plastically deformed polycrystals. This mesoscale structure $(\sim 30-50$ $\mu \mathrm{m})$ has typically not been observed by transmission electron microscopy. Evidence of finer length scales $(\sim 5 \mu \mathrm{m})$ is also often found, as is evident in Fig. 3.

\section{Discussion}

Experimental methods based upon OIM can provide relevant information at the mesoscale concerning GNDs and their spatial distribution. Here we emphasize a few key points: (1) What is directly measured is lattice curvature; the exact distribution, among available dislocations by type, is not recovered. It follows that the method may not be adequate, standing alone, to distinguish between different physical models. Its strength lies in recovering the net distribution at the mesoscale-a scale that has been less-well characterized by transmission electron microscopy. For models that make explicit predictions of lattice curvature, such as finite-element based simulations in conjunction with crystal plasticity, the OIM-based method is capable of providing direct comparisons. (2) Due to the opacity of crystalline materials to electrons, it is not currently possible to obtain the gradient of lattice orientation in the direction perpendicular to the sample surface plane. Thus, only three of the nine components of the GND tensor can be directly measured by the method. When sample preparation can provide an assurance that the orientation gradient is negligible in this third direction, as is the case with some columnar geometries, then estimates of all nine components are possible. (3) Based upon the stated limitations in spatial and angular resolution, it should be possible to resolve the presence of individual dislocations under ideal operating conditions of the FEGSEM. This has indeed been accomplished under optimum conditions near crack tips [11]. Thus in principle, orientation information could be collected at the scale of the dislocation spacing provided that the densities were not too high. However, it is important to remember that in pushing the instrument to such fine scale measurement and angular precision would make it difficult to simultaneously observe the mesoscale structure seen in Fig. 3. Such would require instrument times that exceed typical limits of stability $(\sim 24 \mathrm{~h})$. It would appear that the method is quite versatile in its possibilities for characterization of a large range of length scales of interest to the theory of plasticity. (4) Although the EBSD-based recovery of lattice information has thus far focused upon lattice orientation, it is quite possible that future configurations of the instrument could potentially recover information about the elastic strain. The current generation of charge coupled device (CCD) cameras is typically binned to use as few as 10,000 binned pixels for the purpose of indexing the EBSD patterns for lattice orientation. If the focus plane were positioned such that a much smaller solid angle of the EBSD pattern was captured, and if CCDs of several million pixels were used to capture the diffraction image, it is quite conceivable that elastic strains of the order $10^{-4}$ could be accessible. This 
has not yet been explored, and it is clear that dwell times would be quite large in order to reduce the signal to noise ratio. However, it is likely that such a configuration would be possible in the future. This would enable a more complete evaluation of Eq. (2), although it would still be essential to correct for the image forces that occur due to the presence of the free surface and other effects such as beam heating. (5) The apparent GND density is dependent on the spatial sampling frequency. A simple thought experiment suffices to illustrate the point. If the orientation map has grid spacing significantly smaller than the dislocation spacing, the dislocation density will, in principle, be the same as that of the actual dislocation network. As the grid spacing is increased, "oscillations" in orientation will become undetected and the apparent GND will decrease.

\section{Acknowledgements}

The authors wish to thank The Alcoa Technical Research Center for supplying the specimens and performing the compression tests. This work was supported primarily by the MRSEC program of the National Science Foundation under DMR0079996.

\section{References}

[1] Taylor GI. J Inst Metals 1938;68:307.

[2] Fleck NA, Muller GM, Ashby MF, Hutchinson JW. Acta Metall Mater 1994;42:475.

[3] Sun S, Adams BL, King WE. Phil Mag A 2000;80:9.

[4] Ashby MF. Phil Mag 1970;21:339.

[5] Kroner E. Continuum theory of dislocations and self stresses. Berlin: Springer; 1958.

[6] Nye JF. Acta Metall 1953;1:153.

[7] Sun S, Adams BL, Shet C, Saigal S, King WE. Scripta Mater 1998;39:501.

[8] El-Dasher B, Adams BL, Rollett AD. Scripta Mater., submitted for publication.

[9] Mura T. Micromechanics of defects in solids. The Netherlands: Martinus Nijhoff Publishers; 1987, 338.

[10] Demirel MC, El-Dasher BS, Adams BL, Rollett AD. In: Electron backscatter diffraction in material science. New York: Kluwer Academic; 2000. p. 65.

[11] Crimp MA, Simkin BA. Phil Mag Lett (UK) 2000;80:395. 